\title{
Current Patent Trends Analysis and Research for Intelligent Industrial Robots Industry —based on deburring robot
}

\author{
Shunjun Zhu ${ }^{1, a}$, Jinsong Chen ${ }^{1, b}$,Jing Li ${ }^{1, c}$, Xuexi Zhang ${ }^{2, d}$, Zhiwen Huang ${ }^{2, e}$ \\ ${ }^{1}$ HENGCHENG INTELLIGENCE SOLUTIONS, Guangzhou, Guangdong, China,510060 \\ ${ }^{2}$ Guangdong University of Technology, Guangzhou, Guangdong, China,510060 \\ a sigdear@gzhcis.com, ${ }^{b}$ cjs@gzhcis.com, ${ }^{\circ}$ lijing@gzhcis.com , d864693750@qq.com
}

\begin{abstract}
Keywords: Deburring robot, technical status quo, patent analysis.
Abstract. More than 50\% of intelligent industrial robots used in the automotive field, and the deburring process is an important part of manufacturing technology. Using the patentometrics method for global deburring robot industry related technical patent statistics, makes patent trend analysis and interpretation coverd the following areas: trends of the application, distribution of priority countries, main applicants, technical topics, IPC distribution. The aim of this paper is provide reference for domestic deburring robot industry related enterprise innovation.
\end{abstract}

\section{Introduction}

With the development of intelligent equipment,robot in the industrial manufacturing advantage is more and more significant.The promotion and application of intelligent industrial robots can enhance the automation and intelligence level of industrial manufacturing, reduce the impact of labor cost and demographic dividend reduction, improve the efficiency of quality and reduce cost.This is undoubtedly the most critical breakthrough in intelligent manufacturing.In recent years, China's intelligent robot technology has made great progress in the field.In the whole machine design and manufacture of the robot has accumulated a certain amount of experience, forming a relatively large base of R \& D team.At present, we have mastered the design and manufacture technology of the robot manipulator, the control system hardware and software design technology, the kinematics and the trajectory planning technology.And the production of some of the key components of robot, the development of painting, arc welding, spot welding, assembly, handling and other robots.Of which more than 130 sets of paint robots in more than 20 enterprises of nearly 30 automatic painting production line (station) on the scale of application, arc welding robots have been used in the welding line of automobile manufacturers[1]. More than 50\% of industrial robots used in the automotive field.It is understood that the United States $60 \%$ of industrial robots for automobile production. The world's industrial robots for the automotive industry has reached $37 \%$ of the total amount of industrial parts for industrial robots about 24\%[2].At present, the field of industrial robots are mainly arc welding, spot welding, assembly, handling, painting, testing, palletizing, grinding and polishing and laser processing and other complex operations. While the precision machining parts in the car will have a variety of burrs. With the development of technology and automation, the complexity of the shape of the parts will continue to increase, more and more high precision, the use of increasingly harsh conditions, parts are not allowed to leave a little glitch.If the glitch is not clean, it will directly affect the normal work of precision parts, and even bring the risk of automatic adjustment failure, resulting in the accident ruin.Deburring process is thus an important part of manufacturing technology, its work accounted for a considerable proportion of machining.In the United States, deburring takes 5-10\% of the total man-hours, costs $10-40 \%$ of manufacturing costs, and deburring of precision parts can be as much as $50 \%$.

Guangdong Provincial Science and Technology Project, Item Number: 2015A040405013 
Therefore, the deburring technology research, not only to improve product performance needs, but also reduce costs and improve work efficiency needs. Mechanical parts deburring process technology After years of research, there are a variety of deburring methods.Domestic robot deburring, although coming soon, but has been considered a promising process. There have been two kinds of robots deburring technology:Robot brush deburring and robotic high-pressure jet deburring, is the robot deburring hot research direction [3].

In the era of globalization and knowledge economy, the market competition has been promoted from product manufacturing to $\mathrm{R} \& \mathrm{D}$ innovation, patent enclosure layout to create value levels.Patent has become a competitive weapon in various fields.Europe and the United States and Japan and other developed countries attach great importance to their patent layout as a means to carry out technology enclosures and market range to seize, restricting the development of China's intelligent industrial robot.In order to understand the status quo of deburring intelligent industrial robots, this paper makes use of "Intelligent Industrial Robot Patent Retrieval Database Platform".This paper analyzes and studies the application trend, research organization, research team and technology geographical distribution of the related patents (As of August 2016 public) of deburring robots, which are represented by the brush deburring robots and the high pressure jet deburring robots.For the domestic industry related enterprise development, competition, innovation and provide reference for reference.

\section{Global deburring robot technology patent application trend analysis}

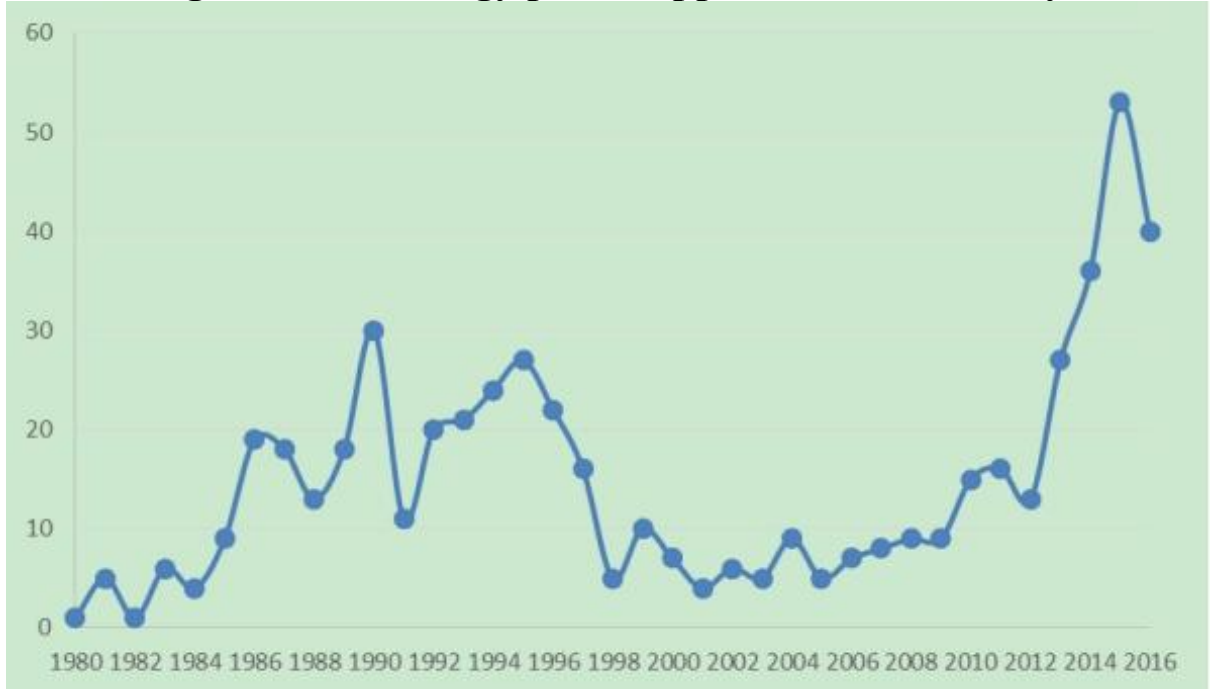

Fig1 Deburring Robot Global Patent Trends (Item)

Figure 1 shows the deburring robot technology global patent applications and the relationship between the application time.It can be seen from the figure the overall trend of this related technology can be divided into four stages.

(1) initial stage of development

During this period, there were few patent applications for deburring robots. In 1958, Prof. Okajima of Kyoto University in Japan published a paper entitled "Burr in the Cutting Process", Then the burr attention and research more and more. 1984, Yokohama University, Japan, Professor Yoshio Kazunori to cutting the most basic elements of cutting direction and cutting tool as the benchmark, cutting burr conducted a more comprehensive and systematic classification of the definition, Which is effective to suppress and reduce the burr generation and laid the theoretical foundation. Therefore, the deburring robots of this stage are intermittently applied and the annual patent filings are in single digits.

(2) the first rapid development stage (1986-1997)

Japan's industrial robots into the practical stage, Japan began to promote the use of industrial robots in various fields, which greatly alleviated the market labor shortage serious social contradictions. Coupled with the Japanese government to adopt a wide range of incentives, these robots received a large number of enterprises welcome. Deburring robots to the rapid development of the period, reflected in the global patent applications, the increase in the amount of relatively stable, from several to gradually increase to dozens of annual. 
(3) Stable development stage (1998-2012)

Deburring robot patent applications have come down, but every year the relevant patent applications, in a stable period of development.

(4) two rapid development stage (2013-present)

In recent years, the patent application of the deburring robot is in rapid progress, which is closely related to the development of the robot industry in China. As China's labor costs continue to rise, the industrial field of "machine substitutions" phenomenon in general, the industrial robot market and industry has gradually developed. Since 2013, China has become the world's largest robot consumer. Consumption of 56,000 robots in 2014, an increase of over 55\%, accounting for the global total consumption of $1 / 4$, compared to 5,800 units in 2006, jumped nearly 10 times. With the industrial transformation and upgrading in the policy, capital, business and other aspects of participation in the context of deburring robot development is ushering in a wave of blowout period.

\section{Global deburring robot technology patent origin country distribution analysis}

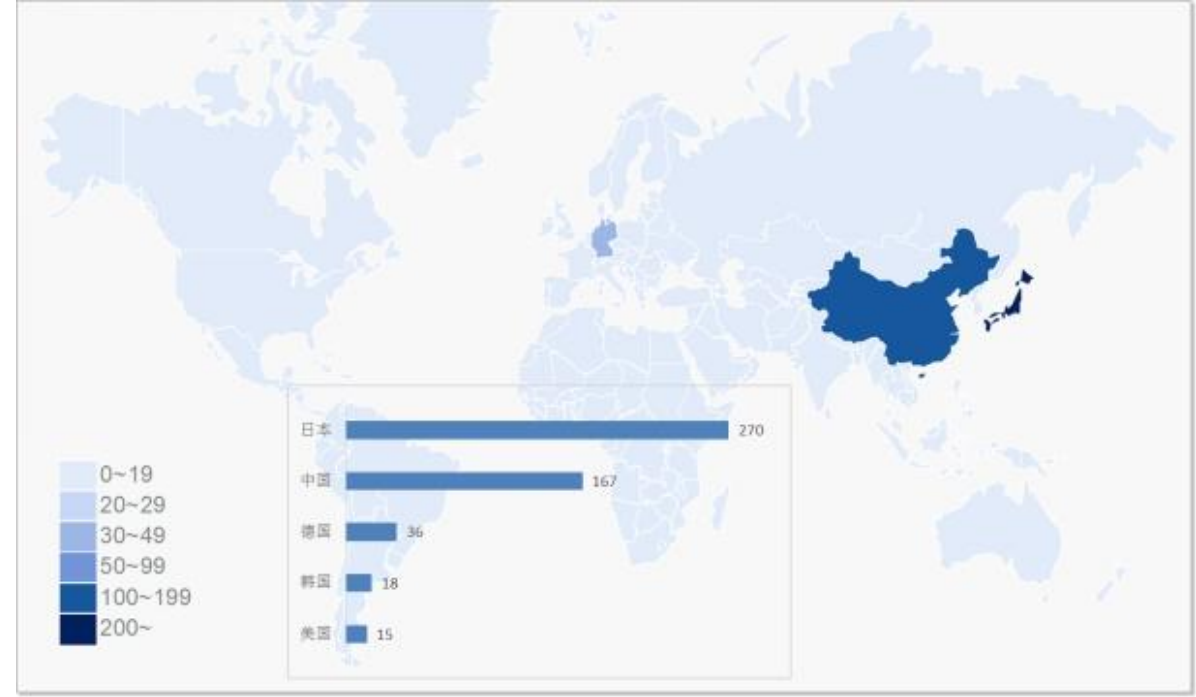

Fig 2 deburring robot patented technology patented country of origin distribution (item)

Figure 2 shows the deburring robot patented technology patented country of origin distribution (the top 5 ranking).

Here, the country / region where the patent application is filed will be the first country to apply for the original country analysis of the patent application for deburring. Patent applications for deburring robots from around the world come from 32 countries. As shown in Figure 2, which ranked application were: 270 in Japan, China 167, 36 in Germany, South Korea 18, the United States 16, 48 other countries and regions.

By analyzing the global patent data of deburring robots, it is found that the original patent applications of deburring robots are mainly from Japan and China. The number of patent applications from these two countries has reached 437, accounting for $80.0 \%$ of the total. Among them, Japan is the largest technology originality, the overall strength of technology and patent density was significantly stronger than other countries and regions. For domestic enterprises, the need to focus on the Japanese applicant for the development of technology and patent application dynamic, to enter the Japanese market need to do a good job ahead of patent layout and risk prevention work. 


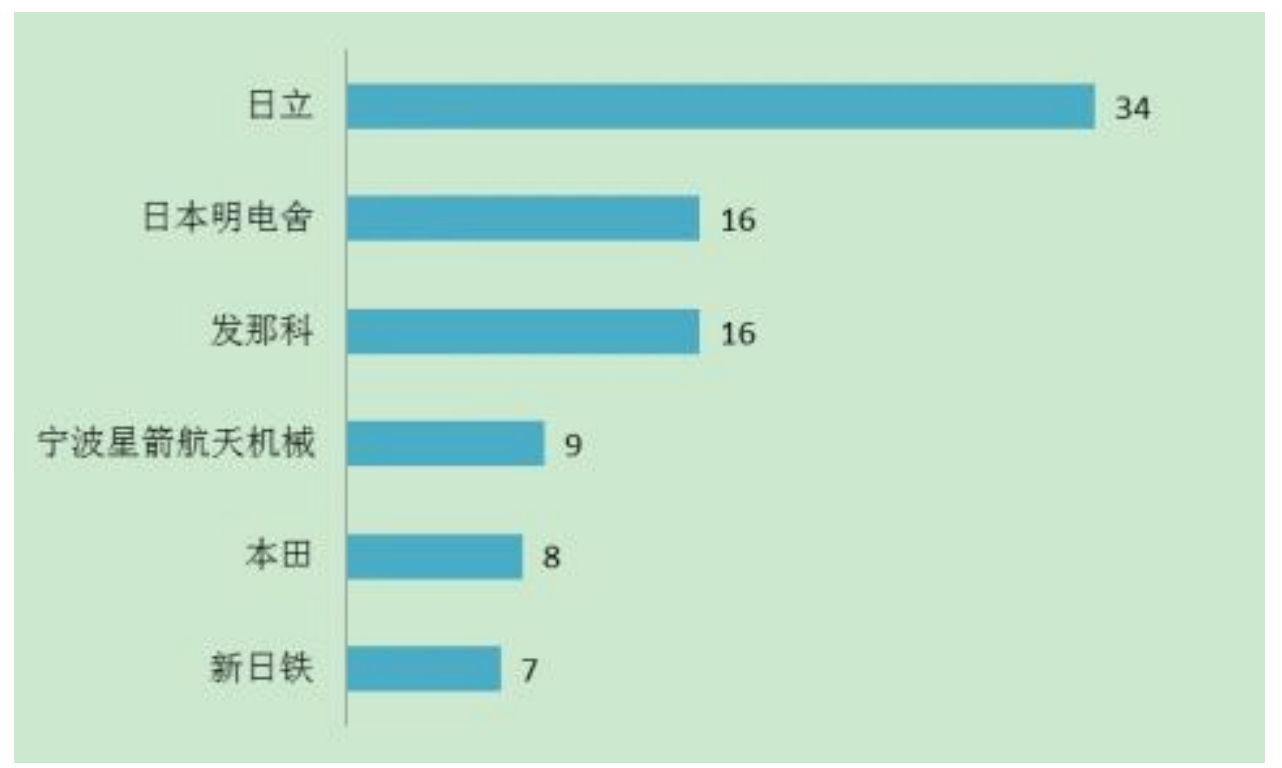

Fig 3 Deburring robot technology The world's major applicants (items)

Figure 3 shows the world's leading applicants for the deburring robot field (top 6 rankings). Among the top applicants are a Chinese company - Ningbo Xingjian Aerospace Machinery Co., Ltd., all other Japanese companies, Japanese enterprises on the industrial robot technology occupies an absolute advantage. Enterprise background source broad, not limited to robot equipment enterprises Fanuc, as well as automotive companies Hitachi, Honda, aerospace companies such as Ningbo Star Arrow. Deburring robots seem to use or production-related businesses have a greater concern on the deburring robot, the market there is a large development needs.

Hitachi, headquartered in Tokyo, Japan, is committed to household appliances, computer products, semiconductors, industrial machinery and other products, is Japan's largest integrated motor manufacturers. Japan's manufacturing industry is second only to Toyota Motor Corporation's second largest manufacturing company in Japan, the industry is also only ranked in Toyota, Japan Post, Japan Telecom, Japan's fourth largest company. The United States, "Fortune" magazine in 2012 selected the world's largest 500 companies ranked No. 38 ranking. In 1988, he was involved in the development of four-legged mobile robots and developed the robot business.

Founded in 1897, the company is headquartered in Tokyo, Japan, the registered capital of 17.07 billion yen (about RMB 1.1 billion yuan), Japan's largest electric appliance manufacturer. It is listed on a market of the Tokyo Stock Exchange and is engaged in the manufacturing and sales of energy, environmental protection, information, communication, industrial system and automobile dynamometer.

Japan FANUC company was established in 1956, is the world's CNC system research, design, manufacture, sales strength of the most powerful enterprises, is a Japanese company specializing in numerical control system. FANUC in 1959 first introduced the electro-hydraulic stepper motor, in the subsequent years to gradually develop and improve the hardware-based open-loop CNC system. Into the 70 's, microelectronics, power electronics, especially in computing technology has been rapid development, FANUC company decided to give up the development of electro-hydraulic stepper motor NC products, on the one hand from GETTES company introduced DC servo motor manufacturing technology. 1976 FANUC company successfully developed numerical control system 5, and then with SIEMENS company jointly developed with the advanced level of numerical control system 7, from then on, FANUC company gradually developed into the world's largest professional CNC system manufacturer. Fanuc NC industrial robots ranked first in global sales accounted for $70 \%$ of the global market share in 2008, the world's first breakthrough in 20 million robots. In 2011, FANUC has installed over 250,000 robots worldwide, making it the world's most influential and most technologically advanced robot and system supplier. 
China Ningbo Xingjian Aerospace Machinery Co., Ltd. is a provincial-level high-tech enterprise, the national "863" plan to bear the unit, set up a provincial high-tech enterprise R \& D Center - Star arrow fluid medium control system research and development center. Since the 80 s of last century with the space enterprises and institutions engaged in the production of stainless steel pipe components, and gradually formed the current can adapt to different high-pressure gas medium and strong corrosive, strong oxidizing media, low temperature medium series of valves, manual and pneumatic cut-off Valve, throttle valve, pressure reducing valve, check valve, safety valve, manual and pneumatic valve, solenoid valve, filter, etc; High-pressure gas for gas, airtight test equipment, including the use of computer-controlled automatic high-pressure gas for gas distribution equipment; Propellant filling system, hydraulic strength test equipment and hydraulic technology, test system equipment; High pressure, special media metal hose and expansion joints; High-pressure non-metallic hose, including rubber, resin, polyurethane, PTFE and ultra-high pressure hose hose; Various pipe fittings, including national (mechanical) standards, aviation standards, aerospace standards, the different standards of the ship, the form of pipe connections; Aviation special fasteners, small parts and high-pressure gas pipeline control system technology based on research and development to meet the different needs of the gas supply vehicles, gas injection vehicles and other products.

Honda is the world's largest motorcycle manufacturer, automobile production and scale also ranked the world's top ten car manufacturers list. Founded in 1948, the company is headquartered in Tokyo, the total number of employees of 18 million people. Now, Honda is a multinational automobile, motorcycle production and sales group. Its products in addition to cars, motorcycles, there are generators, agricultural machinery and other power machinery products. Its products in addition to cars, motorcycles, there are generators, agricultural machinery and other power machinery products.

Nippon Steel Corporation is Japan's multinational companies, Japan's largest steel company, is one of the world's largest steel companies, headquartered in Tokyo, Japan. Nippon Steel Corporation is the predecessor of the 1897 establishment of the official battalion of the Hachiman iron. It is not only the birthplace of Nippon Steel Corporation, but also the birthplace of Japan's steel industry. February 1934 by the official battalion of the Hachiman iron and civilian round West iron, Kashima mine, Fuji Iron, Toyo Steel, Mitsubishi Iron and Steel, Kyushu iron six combined. 1950, divided into Hachiman Steel, Fuji Iron and Steel Corporation and the two iron and steel companies and the Japanese mill firebrick. March 1970, Yahata, Fuji merger of the two companies, the birth of the new Japan Iron and Steel Company, referred to as Nippon Steel, thus becoming the world's largest steel companies.

In the face of the future internationalization and globalization competition, Chinese enterprises need to adjust, should pay attention to the intellectual property management and patent protection strategy, raise the patent application consciousness, collect and select and draw on the technical experience of these strong technology, and accelerate domestic and foreign patents Layout, so as to further enhance the international competitiveness.

Global deburring robot patented technology theme analysis

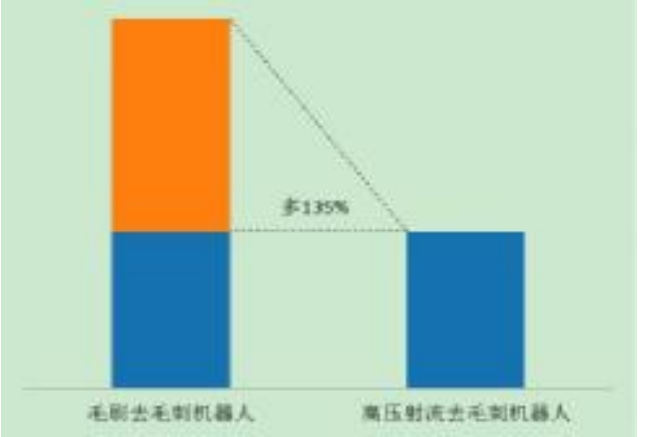

Fig 4 Deburring robot technology topics Comparison (item)

According to the above-mentioned, this paper selects the relatively hot robot brush deburring and robot high-pressure jet deburring technology to carry out research and analysis. It can be seen from Fig. 
4 that the research interest of the brush deburring robot is higher than that of the high pressure water jet deburring robot, and its corresponding patent application quantity is much more than that of the high pressure water jet deburring robot. It may be due to the existence of high-pressure jet deburring technology: high-pressure water system requires high pressure water pressure into the jet kinetic energy consumption, high-pressure system noise and other issues, but also the technology into the current high-pressure jet to The number of patent applications for burr robots is low. The brush deburring robot relatively speaking, more likely to be accepted by the market, so the enthusiasm of research and development into a higher, higher output of patents.

\section{Global deburring robot patented technology composition analysis}

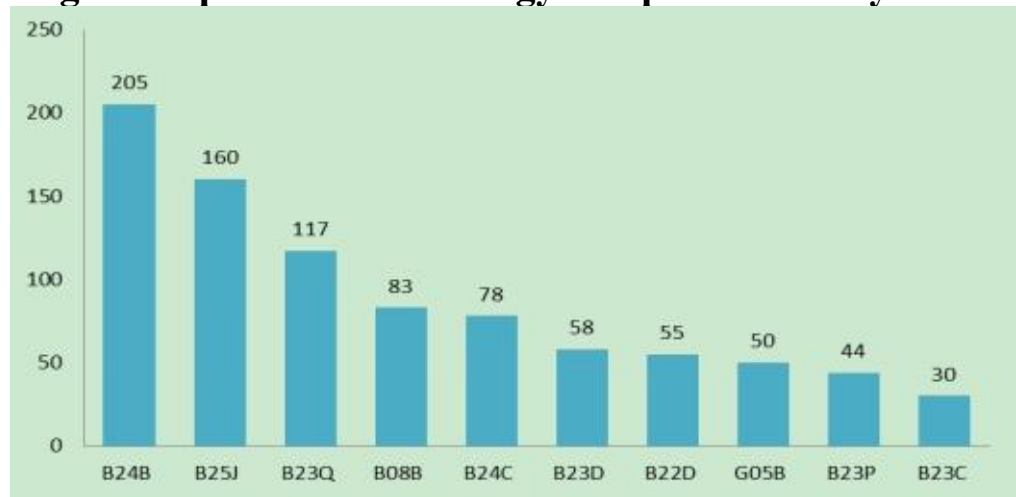

Fig 5 IPC debossing robot technology distribution (item)

As shown in Figure 5 for the deburring robot technology and the relationship between the IPC (the first 10-bit sort). As can be seen from Figure 5, B24B (machine, device or process for grinding or polishing; Repair or adjustment of die wear surfaces; Grinding, polishing or abrasive feed) the largest amount of applications, to 205; The number of patent applications for the second B25J (manipulator; container with controls) and the third B23Q (parts, components or accessories of the machine such as profiling devices or controls) are much larger than the rankings of the next subcategories, There are a considerable number of applications.

The former 10 sorting situation, the deburring robot related technology patent applications mainly in grinding parts, manipulator control, machine control in three directions.

\section{Global patent analysis of deburring robot technology}

Patent size refers to the same invention in different countries to obtain patents or submit the number of patent applications, or the applicant for the same invention to seek patent protection in the number of countries. With the increase in the number of countries seeking protection, patent costs are also increasing, and applicants are more willing to do so for economically valuable, high-tech inventions. At the same time, to apply for a patent to other countries means that the applicant may interpret the invention of international competitiveness, if the final multi-national grant patents that the invention stand the test of many parties, with high technical value. Therefore, the patent family size also reflects the economic importance of the invention and technical importance[4]. Through the number of the same family, but also in combination with other factors to filter out a series of important deburring robot patents, limited space, cited two are as follows.

Patent No. US8006915B: Chinese patent name "ultrasonic water jet", in 2003 by the Canadian VLN Advanced Technology Corporation to apply, the patent Inpadoc with 14 families, The United States, WO and other countries, regions or organizations. Currently 14 patents in Austria, Canada, China, the Czech Republic, some European countries, Spain, Japan, Portugal, the United States are valid. The patent relates to a burr for cutting and cleaning materials, a coating for cleaning and removing the surface, and a high-pressure jet ultrasonic jet for breaking stone. The water jet ejected from the ultrasonic nozzle is pulsed into small water bombs, Applying a water hammer pressure to the target surface, so that the effective impact pressure is much higher than that of the continuous water jet, thereby increasing the cleaning, cutting, cleaning burrs, coating removal and breaking capacity of the apparatus. For a given pressure source, the water hammer pressure is much higher than the stagnation 
pressure of the continuous water jet. Therefore, in order to cut and clean burrs, clean and remove coatings, and break rocks and rock-like substances, ultrasonic water injector can be very low pressure source operation. Ultrasonic water sprayers are therefore more effective, more durable, and less expensive to construct and use than conventional continuous flow water spray systems.

Patent No. US7534077B: Chinese patent name "finishing device" in 2005 by the Japanese FANUCO Co., Ltd., the patent Inpadoc with a total of four, from Europe, China, Japan, the United States, the current four patents in these countries Are effective.

This patent relates to a finishing apparatus for removing a burr on a surface of a workpiece to be machined by a robot by pressing a machining tool against a burr-removing surface of a work to be worked, and then copying the surface, The position data of the surface shape. Further, the error generated by the deflection of the robot is corrected with respect to the position data. The obtained position data is compared with the target shape of the flash removal surface shape obtained from the CAD. obtained position data is compared with the target shape of the flash removal surface shape obtained from the $\mathrm{CAD}$ and the deviation of the normal direction of the surface shape is determined. The occurrence start position and the end position of the burr and the height thereof are obtained from the start position and the end position of the deviation and the size of the deviation. A machining program consisting of a path connecting a termination position and a start position of the flash and a cutting path for removing the flash is formed and executed. Since the flash position is determined and moved to the flash position to remove the flash, the flash removal can be efficiently performed.

\section{Conclusions}

In summary, in order to brush deburring and high-pressure jet deburring deburring robot related technology more layout in Japan, China and other countries, with the rapid pace of China's intelligent manufacturing and construction, intelligent industrial robot industry will become the support of China Economic and sustainable development of the important pillars. Although the current patent applications of deburring robots in China have been ranked behind Japan, but must recognize that the domestic and foreign intelligent industrial robot technology is still a huge gap between the overall level of China's intelligent industrial robot industry is still relatively weak, robot research is still a heavy burden far. In the process of leading the transformation and upgrading of traditional manufacturing industry, China's industrial robots in technological innovation to increase the intensity at the same time, should pay attention to the robot $\mathrm{R} \& \mathrm{D}$, manufacturing and application of the effective link between the use of patents, standards and other technological innovation " Umbrella "in the fierce competition in the market to safeguard their own rights and interests, continue to accumulate competitive advantage.

\section{References}

[1] Gan Shaoning.Strategic emerging industry patent technology trends [M]. Beijing: Intellectual Property Press, 2013: 581.

[2] Lian Lijing. The Importance of Industrial Robots in Automotive Industry [J]. Modern Spare Parts, 2009 (2).

[3] ZHENG Xiu-hong. Research on Deburring Equipment of Robot Brush in Automobile Engine Cylinder Cover Production Line [D]. Guangdong University of Technology.

[4] SUN Tao-tao, TANG Xiao-li, LI Yue.Identification of core patent and its empirical study

[J] .Library and Information Work, 2012 (4): 80-84.

[5] Li,Q.H.,Liu,Y.,\&Wu,S.Z.,et al. Patent value indicators and their structure.J Studies in Science of Science(in Chinese) 2007;25:281-286.

[6] Wu,H.,\&Chang,F.Analysis and solution to the present sitution of patent based on effective patent .J Library and Infoemation Service 2012;55:85-89. 